\title{
Evaluation of demodicosis, nailfold capillaroscopy, and oxidative stress in rosacea: a case-control study
}

\author{
Ezgi Aktaş Karabay, ${ }^{1,2}$,̈zlem Unay Demirel ${ }^{3}$
}

${ }^{1}$ Department of Dermatology and Venereology, Faculty of Medicine, Bahçeşehir University, Istanbul, Turkey ${ }^{2}$ Department of Dermatology and Venereology, Okmeydanı Training and Research Hospital, Istanbul, Turkey ${ }^{3}$ Department of Biochemistry, Faculty of Medicine, Bahçeşehir University, Istanbul, Turkey

Adv Dermatol Allergol 2021; XXXVIII (4): 590-596 DOI: https://doi.org/10.5114/ada.2021.108917

\begin{abstract}
Introduction: Inflammation, immune system disorders, Demodex infestation, neurovascular dysregulation and oxidative stress are thought to be contributory factors in the pathogenesis of rosacea.

Aim: To evaluate the presence of Demodex mites, the morphologic features of the nailfold capillaries, and the systemic oxidative stress status in patients with rosacea.

Material and methods: Thirty-one patients diagnosed with rosacea and 37 healthy age- and gender-matched subjects were included in this prospective case-control study. The presence of Demodex infestation, the findings of nailfold capillaroscopy (NFC), and the status of systemic oxidative stress measured by total oxidant capacity (TOC), total antioxidant capacity (TAC) and oxidative stress index (OSI) were evaluated.

Results: Demodex infestation rates were significantly higher in rosacea patients than in controls $(p=0.001)$. Increases in the diameters of the capillaries and the presence of avascular areas and crossing and abnormal structures were significantly more common in rosacea patients than in the healthy controls $(p<0.01, p=0.016, p=0.02, p<$ 0.001 , respectively), and hairpin structures were significantly less common in rosacea patients than in the controls $(p<0.001)$. The presence of crossing capillaries was positively correlated with higher TOC levels $(p=0.05)$, while abnormal structures were found to be correlated with lower levels of TAC $(p=0.045)$.

Conclusions: Oxidative status and NFC may play diagnostic and prognostic roles in rosacea, which should be confirmed by studies with larger sample sizes.
\end{abstract}

Key words: Demodex, nailfold capillaroscopy, oxidative stress, rosacea.

\section{Introduction}

Rosacea is a chronic inflammatory cutaneous disorder, with remission and exacerbation periods [1]. Flushing, erythema, telangiectasia, oedema, papules, pustules, phymatous changes, and ocular lesions are the signs of the disease $[1,2]$. Various factors are thought to contribute to the pathogenesis of rosacea, including genetic predisposition, immune system disorders, pilosebaceous unit abnormalities, ultraviolet light exposure, Demodex colonization, neurovascular dysregulation, and oxidative stress [1-3]. However, the aetiology of rosacea remains uncertain [2].

Several researchers have investigated the factors that could contribute to the pathogenesis of the disease. Studies evaluating the relationship between Demodex mites and rosacea have mostly indicated a positive relationship [4-6]. Demodex mites are found on normal human skin, but an increased number of mites is considered to play a role in inflammatory and immune reactions during the development of rosacea [5, 6].

Nailfold capillaroscopy (NFC) is an easy, non-invasive technique to assess the microcirculation morphology [7]. NFC is considered a useful tool for the early detection of microvascular changes that could develop in inflammatory conditions. While systemic sclerosis and Raynaud's phenomenon are the two most common diseases for which NFC is performed in determining the diagnosis and prognosis [8], changes in the nailfold capillaries have been identified in several conditions, including autoimmune diseases, rheumatic diseases [9], peripheral arterial obliterative disorders [10], venous insufficiency [11], diabetes mellitus [12], hypertension [13], psoriasis [14], and aging [15]. In a recent study, NFC was performed in rosacea patients, but no specific findings were revealed [16].

\footnotetext{
Address for correspondence: Ezgi Aktaş Karabay Assist. Prof., Department of Dermatology and Venereology, Faculty of Medicine, Bahçeşehir University, Sahrayı Cedit Mahallesi, Batman Sk. No. 66, 34734 Kadıköy, Istanbul, Turkey, phone: +90 5055853561, fax: +90 2123810020, e-mail: ezgiaktasmd@gmail.com Received: 4.10.2019, accepted: 3.03.2020.
} 
The role of oxidative stress in several inflammatory diseases, including rosacea, has also been examined [3, 17-19]. Total antioxidant status (TAC) and total oxidant status (TOC) are the parameters that provide an assessment of global oxidative stress [20, 21].

\section{Aim}

In this study, we aimed to evaluate the presence of Demodex mites, the morphologic features of the nailfold capillaries as an indicator of microvascular changes, and the systemic oxidative stress status by measuring TOC, TAC, and the oxidative stress index (OSI) of patients with rosacea, which may reflect the key mechanisms of the rosacea pathogenesis: inflammation, immune system disorder, and vascular changes.

\section{Material and methods}

The study was reviewed and approved by the local ethics committee (Protocol number: 22481095-020-1259; Date of approval: 26/06/2018), and all the individual participants gave written informed consent. The study was carried out according to the principles expressed in the Declaration of Helsinki.

A prospective case-control study was planned to investigate the presence of Demodex infestation and determine the findings of NFC and the status of oxidative stress in patients with rosacea.

\section{Patient selection}

Thirty-one patients diagnosed with rosacea by clinical examination were recruited from the dermatology outpatient clinic. For comparison, 37 healthy age- and gender-matched controls with no evidence of rosacea or any other inflammatory skin disease were recruited from among hospital staff volunteers.

Rosacea is classified as erythematotelangiectatic rosacea (ETR), papulopustular rosacea (PPR), phymatous rosacea, ocular rosacea, and variant granulomatous rosacea, according to the American National Rosacea Society (NRS) Expert Committee classification and staging system, which also acts as a diagnostic tool [1].

Only those with normal body mass index (BMI) $\left(18.5-25 \mathrm{~kg} / \mathrm{m}^{2}\right)$ were included. For each patient, the age, sex, clinical diagnosis, and symptoms were recorded. None of the subjects were under any topical treatment, including moisturizers, within the last 2 months. Subjects with a history of any ablative facial treatments (e.g., peels and laser procedures) in the prior 6 months were also excluded. Patients with a history of any systemic treatment, including corticosteroids, vasoconstrictive or vasodilator agents, non-steroidal antiinflammatory drugs, and immunosuppressants, within 6 months of the study and any systemic diseases, such as hypertension, diabetes mellitus, cardiovascular dis- ease, autoimmune diseases, peripheral vascular diseases (e.g., including scleroderma, Reynaud's phenomenon, dermatomyositis, or concomitant inflammatory or infectious diseases) were excluded. Currently pregnant or lactating females, smokers, and patients with a history or current diagnosis of malignancy were also excluded. The patients who recently had a manicure or who had removed a cuticle in the last 2 months or had a history of trauma involving the nail bed and proximal nail fold in the last 6 months were excluded, since cuticle trauma could affect the evaluation of the nailfold capillaries by NFC.

Data on the baseline demographics, clinical characteristics, and test results were obtained on the same day.

\section{Evaluation of Demodex infestation}

The presence of demodicosis was evaluated by the same dermatologist in each patient. Cyanoacrylate glue standardized skin surface biopsies (SSSBs) were taken and microscopically examined for mites in both the patient and control groups. Two samples were taken for mite examination from the erythematous/inflammatory lesions of the disease on the face. Samples were collected from cheeks and frontal areas in the controls. A slide covered with cyanoacrylate glue and a marked square was pressed against the skin surface. After $30 \mathrm{~s}$, the slide was removed and the samples were collected. The preparation was examined under a light microscope at 40x and 100x magnification. The result was considered positive when there were more than five Demodex mites in a 1- $\mathrm{cm}^{2}$ area [22].

\section{Performing nailfold capillaroscopy}

NFC was performed by a single dermatologist for all subjects. MoleMax HD digital dermoscopy programme was used for the NFC examination. All of the digits of both hands except the thumbs were evaluated during the examination using transparent oily medium (mineral oil) drops on the area to be examined so that the skin would be more translucent and less irregular. The morphologic characteristics of the nailfold capillaries were identified as follows: (1) avascular areas, (2) increases in the diameters of the capillaries, (3) microhaemorrhages, (4) hairpin capillaries, (5) crossing capillaries, (6) tortuous capillaries, and (7) abnormal structures (none of the morphologies that have been identified) (Figure 1).

\section{Assessment of oxidative capacity}

Serum TOC and TAC were measured in all subjects using fasting venous blood samples. The venous blood samples were drawn from the participants between 9:00 and 11:00 a.m. following a 12-hour fasting period. TOC and TAC were spectrophotometrically measured using Rel Assay commercial kits (Rel Assay Kit Diagnostics, Turkey). OSI was calculated as the ratio of TOC to TAC. 

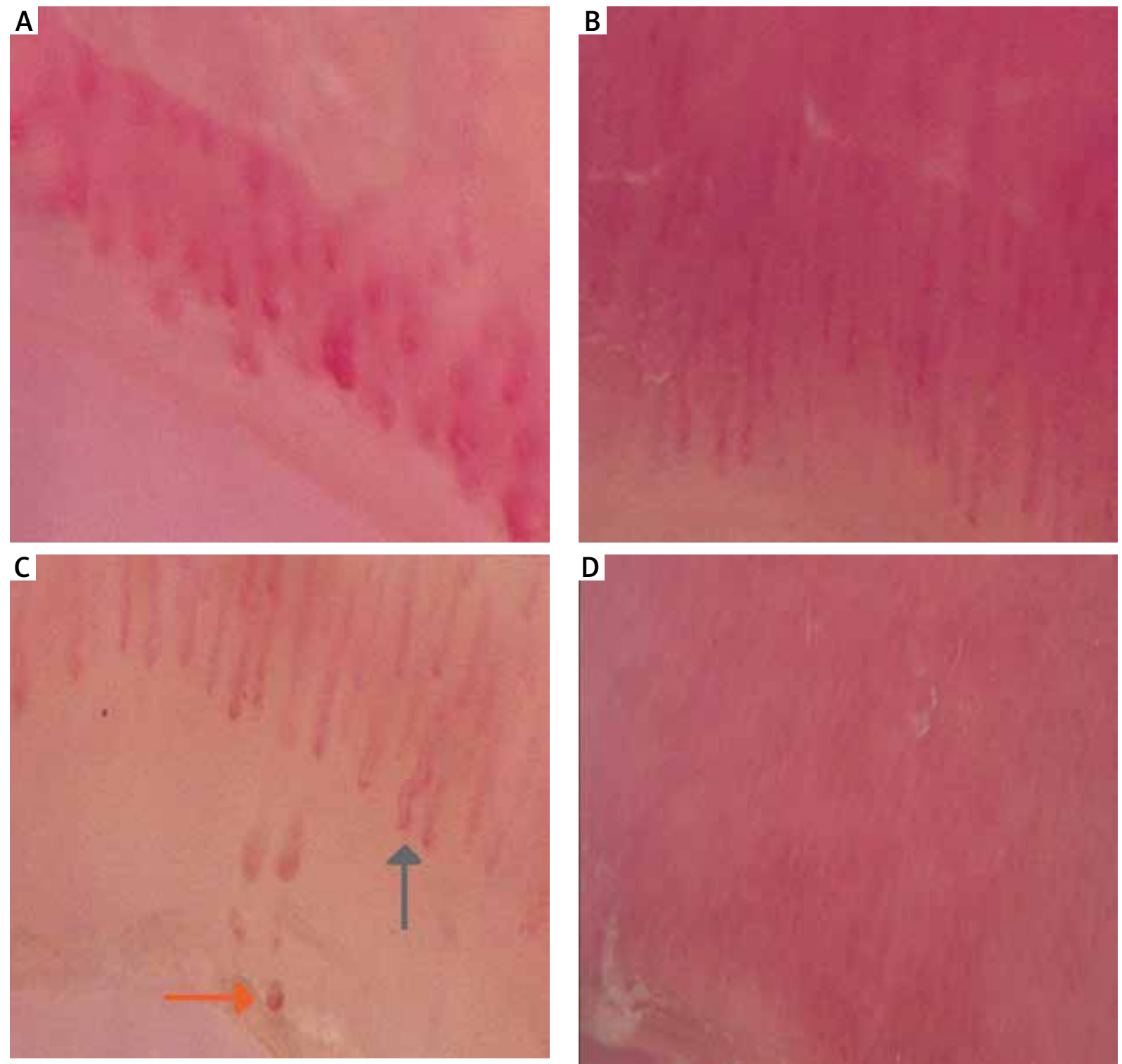

Figure 1. Nailfold capillaroscopy. A - Increase in the diameters of the capillaries. B - Hairpin structures. C - Hairpin structures, one slightly tortuous capillary (green arrow), microhaemorrhage (orange arrow). D - Absence in capillaries

\section{Statistical analysis}

The Number Cruncher Statistical System 2007 (NCSS; Kaysville, Utah, USA) program was used for the statistical analysis. The data were expressed as mean \pm standard deviation, median (first quartile, third quartile), count, and percentages. In the analysis of normally distributed variables, an independent samples t-test was applied to examine the differences between the two groups. The differences between two independent groups were examined using the Mann Whitney $U$ test for non-normally distributed variables. A one-way ANOVA, with Bonferroni corrected post-hoc tests, was used to compare normally distributed variables between three or more groups. A Kruskal-Wallis test and Dunn-Bonferroni post-hoc tests were used to compare non-normally distributed variables between three or more groups. A Pearson $\chi^{2}$ test, Fisher's exact test, and Fisher-Freeman-Halton test were used to compare categorical variables. The association between variables was evaluated by Spearman's rank correlation coefficient. A $p$-value of $<0.05$ was considered statistically significant.

\section{Results}

A total of 31 patients with rosacea and 37 healthy control subjects were included in the study. No significant differences were observed in terms of gender or age between the patients with rosacea and the healthy controls $(p>0.05)$ (Table 1). None of the subjects had any accompanying disease or smoking habit. The rosacea group 
consisted of 21 (30.9\%) patients with ETR and 10 (14.7\%) patients with PPR. The duration of the disease ranged from 6 to 120 months (mean: $25.42 \pm 24.53$ ).

Sixteen of the 31 (51.6\%) patients had a Demodex infestation, while none of the $37(0 \%)$ healthy controls had an infestation. The Demodex infestation rates were significantly higher in patients than in the controls $(p=$ 0.001) (Table 1). When patients with rosacea were evaluated according to disease subtype, the Demodex infestation was more common in the PPR group than in the ETR group ( $p=0.018)$.

The most common morphologic pattern of nailfold capillaries in rosacea patients was avascular areas ( $n=23,74.2 \%)$, followed by crossing capillaries ( $n=15$, $48.4 \%$ ), tortuous capillaries ( $n=14,45.2 \%$ ), abnormal structures ( $n=14,45.2 \%)$, hairpin capillaries ( $n=11$, $35.5 \%)$, microhaemorrhages ( $n=8,25.8 \%)$, and increases in the diameters of the capillaries $(n=5,16.1 \%)$.

The most common morphologic pattern of the nailfold capillaries in the healthy subjects was hairpin capillaries ( $n=29,78.4 \%$ ), followed by tortuous capillaries ( $n=11,29.7 \%)$, crossing capillaries $(n=8,21.6 \%)$, microhaemorrhages ( $n=6,16.2 \%)$, and avascular areas $(n=5,13.5 \%)$, while none of them had increases in the abnormal structures $(n=0)$ or the diameters of the capillaries.

The presence of avascular areas, increases in the diameters of the capillary structures, and crossing and abnormal structures were significantly more common in the rosacea patients than in the healthy controls $(p<0.01$, $p=0.016, p=0.02, p<0.001$, respectively).

The presence of hairpin structures was more common in the controls than in the rosacea patients ( $p<$ 0.001).

No significant differences were observed in the levels of TAC or TOC between the rosacea group and the control group (4.36 vs. 4.36, $p=0.522,7.22$ vs. $5, p=0.379$, respectively). The OSI values also did not show any significant difference between the two groups (1.39 vs. 1.09, $p=0.772)$.

Table 1. Comparison of demographic data and the presence of Demodex infestation, NFC findings and oxidative stress status between patients with acne vulgaris, rosacea, seborrheic dermatitis and controls

\begin{tabular}{|c|c|c|c|}
\hline Parameter & Healthy controls & Rosacea patients & $P$-value \\
\hline Age [year]; mean \pm SD & $34.68 \pm 7.40$ & $36.55 \pm 11.88$ & ${ }^{\dagger} 0.431$ \\
\hline \multicolumn{4}{|l|}{ Gender; $n(\%)$} \\
\hline Female & $24(64.9)$ & $22(71)$ & ${ }^{\ddagger} 0.592$ \\
\hline Male & $13(35.1)$ & $9(29)$ & \\
\hline $\mathrm{BMI}$; mean \pm SD & $22.91 \pm 1.32$ & $22.97 \pm 1.57$ & ${ }^{\dagger} 0.877$ \\
\hline \multicolumn{4}{|l|}{ Fitzpatrick skin type; $n$ (\%) } \\
\hline 1 & $1(2.7)$ & $8(25.8)$ & $\S 0.007^{* *}$ \\
\hline 2 & $15(40.5)$ & $15(48.4)$ & \\
\hline 3 & $17(45.9)$ & $5(16.1)$ & \\
\hline 4 & $4(10.8)$ & $3(9.7)$ & \\
\hline Demodex infestation; $n(\%)$ & $0(0)$ & $16(51.6)$ & ${ }^{\ddagger}<0.001^{* *}$ \\
\hline \multicolumn{4}{|l|}{ NFC findings; $n(\%)$} \\
\hline Avascular area & $5(13.5)$ & $23(74.2)$ & ${ }^{\ddagger}<0.001^{* *}$ \\
\hline Increase in the diameter of the capillaries & $0(0)$ & $5(16.1)$ & $" 0.016^{*}$ \\
\hline Microhaemorrhage & $6(16.2)$ & $8(25.8)$ & ${ }^{\ddagger} 0.330$ \\
\hline Hairpin structures & $29(78.4)$ & $11(35.5)$ & ${ }^{\ddagger}<0.001^{\star \star}$ \\
\hline Tortuous structures & $11(29.7)$ & $14(45.2)$ & $\ddagger_{0} 0.189$ \\
\hline Crossing structures & $8(21.6)$ & $15(48.4)$ & ${ }^{\ddagger} 0.020^{*}$ \\
\hline Abnormal structures & $0(0)$ & $14(45.2)$ & ${ }^{\ddagger}<0.001^{* *}$ \\
\hline \multicolumn{4}{|l|}{ Oxidative stress; min.-max. (med.): } \\
\hline TOC & $5(1.44,9.33)$ & $7.22(2.33,10.44)$ & "0.379 \\
\hline TAC & $4.36(1.45,7.27)$ & $4.36(1.45,13.09)$ & 0.522 \\
\hline OSI & $1.09(0.53,3.37)$ & $1.39(0.28,5.59)$ & '0.772 - l \\
\hline
\end{tabular}

${ }^{t}$ Independent groups t-test, ${ }^{\ddagger}$ Pearson $\chi^{2}$ test, ${ }^{\S}$ Fisher-Freeman-Halton exact test, "Fisher's exact test, "Mann-Whitney U test; ${ }^{*} p<0.05$, ${ }^{* *} p<0.01$. BMI - body mass index, TAC - total antioxidant capacity, TOC - total oxidant capacity, OSI - oxidative stress index. 
The evaluations for Demodex infestation, NFC findings, and TAC, TOC, and OSI values according to disease subtypes (ETR and PPR) are shown in Table 2.

A positive correlation was noted between the presence of crossing capillaries and higher TOC levels ( $p=$ 0.05). The abnormal structures discerned from NFC were found to be correlated with lower levels of TAC $(p=0.045)$.

\section{Discussion}

In this study, the presence of a Demodex infestation was demonstrated to be more common in the patients with rosacea than in the healthy controls. Furthermore, some of the morphologic capillary patterns, namely, avascular areas, increases in the diameters of the capillaries, crossing capillaries, and abnormal structures were shown to be more common in the rosacea patients. In addition, abnormal structures in the nailfold capillaries were found to be correlated with lower levels of TAC, while the presence of crossing capillaries was correlated with higher levels of TOC. However, no difference was observed in terms of the values of TAC, TOC, and OSI between the two groups.
Chronic inflammation is the main contributor to the development of rosacea. Immune responses to Demodex mites, the dysregulation of the immune system, vascular changes, and oxidative tissue damage are the factors associated with inflammation [3, 19, 23, 24].

Recently, many studies have been conducted examining the relationship between Demodex mites and rosacea, mostly demonstrating a positive relationship. Demodex mites may contribute to the pathogenesis of rosacea in several ways. The blockage of hair follicles and sebaceous glands by an increased number of mites may result in cutaneous barrier disruption and tissue damage. Subsequent increases in TLR expression, chitin mite exoskeletons and releases of internal mite contents, including bacterial antigens, may trigger an inflammatory reaction and also result in an immune response followed by neutrophil and macrophage activation [2, 25-27].

NFC is a non-invasive imaging technique that provides an assessment of peripheral microcirculation by direct visualization of the dermal papillary capillaries [28]. In some of the diseases that are accompanied by vascular damage, changes in the capillaries may be observed even before the clinical symptoms occur [29].

Table 2. Subgroup comparisons in terms of demographic data and the presence of Demodex infestation, NFC findings and oxidative stress status

\begin{tabular}{|c|c|c|c|c|}
\hline Parameter & Healthy controls & ETR & PPR & $P$-value \\
\hline Age [years]; median (Q1, Q3) & $33(30,38)$ & $33(28,40)$ & $38.5(29,40)$ & ${ }^{\dagger+} 0.616$ \\
\hline \multicolumn{5}{|l|}{ Gender; $n(\%)$ : } \\
\hline Female & $24(64.9)$ & $17(81)$ & $5(50)$ & ${ }^{\ddagger} 0.197$ \\
\hline Male & $13(35.1)$ & $4(19)$ & $5(50)$ & \\
\hline BMI; median (Q1, Q3) & $23.1(21.8,23.8)$ & $23.6(21.3,24.1)$ & $23.55(23.2,24.6)$ & ${ }^{+1} 0.508$ \\
\hline \multicolumn{5}{|l|}{ Fitzpatrick skin type; $n$ (\%): } \\
\hline 1 & $1(2.7)$ & $6(28.6)$ & $2(20)$ & $\S 0.016^{\star}$ \\
\hline 2 & $15(40.5)$ & $11(52.4)$ & $4(30)$ & \\
\hline 3 & $17(45.9)$ & $2(9.5)$ & $3(30)$ & \\
\hline 4 & $4(10.8)$ & $2(9.5)$ & $1(10)$ & \\
\hline Demodicosis; $n$ (\%) & $0(0)$ & $7(33.3)$ & $9(90)$ & $\S<0.001^{\star *}$ \\
\hline Avascular area; $n(\%)$ & $5(13.5)$ & $13(61.9)$ & $10(100)$ & ${ }^{\ddagger}<0.001^{\star \star}$ \\
\hline Increase in the diameters of the capillaries; $n$ (\%) & $0(0)$ & $4(19)$ & $1(10)$ & $\S_{0.017^{*}}$ \\
\hline Microhaemorrhage; $n(\%)$ & $6(16.2)$ & $4(19)$ & $4(40)$ & $\S 0.317$ \\
\hline Hairpin structures; $n$ (\%) & $29(78.4)$ & $7(33.3)$ & $4(40)$ & ${ }^{\ddagger} 0.002^{\star \star}$ \\
\hline Tortuous structures; $n(\%)$ & $11(29.7)$ & $10(47.6)$ & $4(40)$ & $\S 0.393$ \\
\hline Crossing structures; $n(\%)$ & $8(21.6)$ & $15(71.4)$ & $0(0)$ & ${ }^{\ddagger}<0.001^{\star *}$ \\
\hline Abnormal structures; $n(\%)$ & $0(0)$ & $9(42.9)$ & $5(50)$ & $\S<0.001^{\star \star}$ \\
\hline TAC; median (Q1, Q3) & $4.36(1.45,7.27)$ & $4.36(1.45,13.09)$ & $2.18(1.45,10.18)$ & ${ }^{+1} 0.693$ \\
\hline TOC; median (Q1, Q3) & $5(1.44,9.33)$ & $6.67(2.33,10.11)$ & $8.62(3.11,13.11)$ & ${ }^{+1} 0.556$ \\
\hline OSI; median (Q1, Q3) & $1.09(0.53,3.37)$ & $0.91(0.33,4.06)$ & $4.02(0.28,7.2)$ & ${ }^{+1+} 0.492$ \\
\hline
\end{tabular}

${ }^{*}$ Pearson $\chi^{2}$ test, ${ }^{8}$ Fisher-Freeman-Halton exact test, ${ }^{+1}$ Kruskal-Wallis test, ${ }^{*} p<0.05,{ }^{* *} p<0.01,01-$ first quartile, Q3 - third quartile, BMI-body mass index, ETR - erythematotelangiectatic rosacea, PPR - papulopustular rosacea, TAC - total antioxidant capacity, TOC - total oxidant capacity, OSI - oxidative stress index. 
Moreover, abnormalities in the capillary structure have been demonstrated to be indicators of the severity and prognosis of certain diseases. Systemic sclerosis, which is a disease for which NFC is commonly performed, a correlation was shown between the decreased capillary density and the severity of pulmonary arterial hypertension [30]. While the use of NFC in the assessment of microvascular involvement in rheumatologic diseases has been well established, this technique was recently reported to be a useful tool in various other diseases, including arterial hypertension, diabetes mellitus, metabolic syndrome, and cardiac syndrome X [31-33]. However, little is known about the changes in microcirculation in patients with rosacea. As far as we know, one study (of 8 rosacea patients) has been conducted in which a specific capillaroscopic pattern or capillary morphological changes could not be detected [16].

Oxidative stress contributes to the development of rosacea through the activation of neutrophils that produce reactive oxygen species (ROS), oxidative modification of proteins and lipids by ROS, alterations in lipid balance, antimicrobial peptides (cathelicidin LL-37), cytokines, and other inflammatory mediators (IL-1 and TNF- $\alpha$ ) [19, 34]. Increased oxidative stress manifesting as higher levels of TOC and OSI and lower levels of TAC has been reported in patients with rosacea in recent studies [3, 17-19].

A growing body of evidence suggests that rosacea is associated with systemic diseases. A shared pathophysiologic link between the systemic diseases and rosacea has not been clarified, but chronic inflammation is the most prominent underlying mechanism among the metabolic, immune, and endocrine changes [35]. Cardiovascular diseases and gastrointestinal, neurologic, and psychiatric disorders are the main conditions that are reported to accompany rosacea and chronic inflammation, and increased oxidative stress is thought to contribute to the pathogenesis of each disease $[3,35]$.

It is known that inflammation is considered the key factor in the pathogenesis of rosacea. Inflammation is also associated with other factors, including vascular changes and oxidative stress. In this study, we hypothesized that there might be a correlation between the presence of Demodex infestation, which may act as a trigger for the inflammatory and immune reactions, the vascular changes in nailfold capillaries, and increased oxidative stress, as a result of inflammation and contributors to inflammation in patients with rosacea.

In the present study, patients with rosacea had significantly higher rates of Demodex infestations than the healthy controls, which is consistent with the extant literature. In NFC examinations, the presence of avascular areas, increases in the diameters of the capillary structures, and the presence of crossing and abnormal structures were significantly more common in the rosacea patients than in the healthy controls. Abnormal structures and increases in the diameters of the capillaries were not detected in the NFC examinations of any of the healthy subjects, which suggests that these two features might be specific to rosacea. Moreover, a positive correlation between the presence of crossing capillaries and higher TOC levels was observed, and a correlation between abnormal structures and lower levels of TAC was observed. Taken together, it may suggest that abnormal structures in the nailfold capillaries of rosacea patients associated with lower oxidative capacity may be a predictor of comorbidities in rosacea, which might be a subject of another study. However, no correlation was found between the oxidative stress markers and the occurrence of rosacea.

The small sample size is an important limitation of the study. The variations of subgroup numbers are also a limitation.

\section{Conclusions}

Demodex infestations and some NFC findings, such as the presence of avascular areas, increases in the diameters of the capillaries structures, crossing capillaries and abnormal structures, have been demonstrated to be significantly more common in rosacea patients. Among them, abnormal structures may require caution for two reasons: (1) none of the healthy subjects had abnormal capillaries in the NFC examination, and (2) abnormal structures in nailfold capillaries were found to be associated with lower antioxidant capacities. We believe that studies with larger sample sizes are necessary to make conclusions on the diagnostic and prognostic role of the oxidative status and NFC in rosacea.

\section{Conflict of interest}

The authors declare no conflict of interest.

\section{References}

1. Wilkin J, Dahl M, Detmar M, et al. Standard grading system for rosacea: report of the National Rosacea Society Expert Committee on the classification and staging of rosacea. J Am Acad Dermatol 2004; 50: 907-12.

2. Crawford GH, Pelle MT, James WD. Rosacea: I. Etiology, pathogenesis, and subtype classification. J Am Acad Dermatol 2004; 51: 327-41.

3. Falay Gur T, Erdemir AV, Gurel MS, et al. The investigation of the relationships of demodex density with inflammatory response and oxidative stress in rosacea. Arch Dermatol Res 2018; 310: 759-67.

4. Casas C, Paul C, Lahfa M, et al. Quantification of Demodex folliculorum by PCR in rosacea and its relationship to skin innate immune activation. Exp Dermatol 2012; 21: 906-10.

5. Chang YS, Huang YC. Role of Demodex mite infestation in rosacea: a systematic review and meta-analysis. J Am Acad Dermatol 2017; 77: 441-7.

6. Moran EM, Foley R, Powell FC. Demodex and rosacea revisited. Clin Dermatol 2017; 35: 195-200. 
7. Cutolo M, Sulli A, Smith V. How to perform and interpret capillaroscopy. Best Pract Res Clin Rheumatol 2013; 27 237-48.

8. van den Hoogen F, Khanna D, Fransen J, et al. 2013 classification criteria for systemic sclerosis: an American College of Rheumatology/European League Against Rheumatism Collaborative Initiative. Ann Rheum Dis 2013; 72: 1747-55.

9. Cutolo M, Melsens K, Wijnant S, et al. Nailfold capillaroscopy in systemic lupus erythematosus: a systematic review and critical appraisal Autoimmun Rev 2018; 17: 344-52.

10. Lambova SN. The place of nailfold capillaroscopy among instrumental methods for assessment of some peripheral ischaemic syndromes in rheumatology. Folia Med 2016; 58: 77-88.

11. Aguilar Peralta GR, Arévalo Gardoqui J, Llamas Macías FJ, et al. Clinical and capillaroscopic evaluation in the treatment of chronic venous insufficiency with Ruscus aculeatus, hesperidin methylchalcone and ascorbic acid in venous insufficiency treatment of ambulatory patients. Int Angiol 2007; 26: 378-84.

12. Maldonado G, Guerrero R, Paredes C, Rios C. Nailfold capillaroscopy in diabetes mellitus. Microvas Res 2017; 112: 41-6.

13. Junqueira CLC, Magalhães MEC, Brandão AA, et al. Microcirculation and biomarkers in patients with resistant or mild to-moderate hypertension: a cross-sectional study. Hypertens Res 2018; 41: 515-23.

14. Ribeiro CF, Siqueira EB, Holler AP, et al. Periungual capillaroscopy in psoriasis. An Bras Dermatol 2012; 87: 550-3.

15. Li L, Mac-Mary S, Sainthillier JM, et al. Age-related changes of the cutaneous microcirculation in vivo. Gerontology 2006 52: 142-53.

16. Fonseca GP, Brenner FM, de Souza Muller C, Wojcik AL. Nailfold capillaroscopy as a diagnostic and prognostic method in rosacea. An Bras Dermatol 2011; 86: 87-90.

17. Takci Z, Bilgili SG, Karadag AS, et al. Decreased serum paraoxonase and arylesterase activities in patients with rosacea. J Eur Acad Dermatol Venereol 2015; 29: 367-70.

18. Tisma VS, Basta-Juzbasic A, Jaganjac M, et al. Oxidative stress and ferritin expression in the skin of patients with rosacea. J Am Acad Dermatol 2009; 60: 270-6.

19. Erdogan HK, Bulur I, Kocaturk E, et al. Advanced oxidation protein products and serum total oxidant/antioxidant status levels in rosacea. Adv Dermatol Allergol 2018; 35: 304-8.

20. Erel O. A new automated colorimetric method for measuring total oxidant status. Clin Biochem 2005; 38: 1103-11.

21. Erel O. A novel automated direct measurement method for total antioxidant capacity using a new generation, more stable ABTS radical cation. Clin Biochem 2004; 37: 277-85.

22. Eser A, Erpolat S, Kaygusuz I, et al. Investigation of Demodex folliculorum frequency in patients with polycystic ovary syndrome. An Bras Dermatol 2017; 92: 807-10.

23. Gerber PA, Buhren BA, Steinhoff M, Homey B. Rosacea: the cytokine and chemokine network. J Investig Dermatol Symp Proc 2011; 15: 40-7.

24. Holmes AD. Potential role of microorganisms in the pathogenesis of rosacea. J Am Acad Dermatol 2013; 69: 1025-32.

25. Koller B, Muller-Wiefel AS, Rupec R, et al. Chitin modulates innate immune responses of keratinocytes. PLoS One 2011; 6: e16594.

26. Lee JB, Moon J, Moon KR, et al. Epidemiological and clinical features of rosacea in Korea: a multicenter cross-sectional study. J Dermatol 2018; 45: 546-53.

27. Yamasaki K, Kanada K, Macleod DT, et al. TLR2 expression is increased in rosacea and stimulates enhanced serine pro- tease production by keratinocytes. J Invest Dermatol 2011; 131: 688-97.

28. Karbalaie A, Emrani Z, Fatemi A, et al. Practical issues in assessing nailfold capillaroscopic images: a summary. Clin Rheumatol 2019; 38: 2343-54.

29. Martínez-Sales V, Vila V, Ricart JM, et al. Increased circulating endothelial cells and microparticles in patients with psoriasis. Clin Hemorheol Microcirc 2015; 60: 283-90.

30. Hofstee HM, Vonk Noordegraaf A, Voskuyl AE, et al. Nailfold capillary density is associated with the presence and severity of pulmonary arterial hypertension in systemic sclerosis. Ann Rheum Dis 2009; 68: 191-5.

31. Pasqui AL, Puccetti L, Di Renzo M, et al. Structural and functional abnormality of systemic microvessels in cardiac syndrome X. Nutr Metab Cardiovasc Dis 2005; 15: 56-64.

32. Gallucci F, Russo R, Buono R, et al. Indications and results of videocapillaroscopy in clinical practice. Adv Med Sci 2008; 53: $149-50$.

33. Francischetti EA, Tibirica E, da Silva EG, et al. Skin capillary density and microvascular reactivity in obese subjects with and without metabolic syndrome. Microvasc Res 2011; 81: 325-30.

34. Jones DA. Rosacea, reactive oxygen species, and azelaic acid. J Clin Aesthet Dermatol 2009; 2: 26-30.

35. Haber R, El Gemayel M. Comorbidities in rosacea: a systematic review and update. J Am Acad Dermatol 2018; 78 : 786-92. 